\title{
Germanica
}

\section{Recherche d'identité et théâtre politique Le choix du genre chez Peter Weiss dans les années soixante et soixante-dix}

Identitätssuche und politisches Theater.

Die Wahl des Genres bei Peter Weiss in den sechziger und siebziger Jahren

\section{Karl Heinz Götze}

\section{OpenEdition Journals}

Édition électronique

URL : http://journals.openedition.org/germanica/1969

DOI : 10.4000/germanica. 1969

ISSN : 2107-0784

\section{Éditeur}

Université de Lille

\section{Édition imprimée}

Date de publication : 30 juin 1996

Pagination : $97-114$

ISBN : 9782098426320

ISSN : 0984-2632

\section{Référence électronique}

Karl Heinz Götze, «Recherche d'identité et théâtre politique

Le choix du genre chez Peter Weiss dans les années soixante et soixante-dix », Germanica [En ligne],

18 | 1996, mis en ligne le 13 juin 2013, consulté le 06 octobre 2020. URL : http://

journals.openedition.org/germanica/1969; DOI : https://doi.org/10.4000/germanica.1969

Ce document a été généré automatiquement le 6 octobre 2020.

(c) Tous droits réservés 


\title{
Recherche d'identité et théâtre politique
}

\section{Le choix du genre chez Peter Weiss dans les années soixante et soixante-dix}

Identitätssuche und politisches Theater. Die Wahl des Genres bei Peter Weiss in den sechziger und siebziger Jahren

\author{
Karl Heinz Götze
}

Parmi les auteurs majeurs de langue allemande du vingtième siècle, aucun n'a travaillé dans des domaines aussi divers que Peter Weiss. Il débuta dans la peinture. Du début des années trente jusqu'à la fin des années quarante, il se considéra avant tout comme un peintre et s'il privilégia plus tard d'autres moyens artistiques, il travailla, au moins jusqu'aux «Collages » qui accompagnent Abschied von den Eltern (1962), comme artiste peintre. Son œuvre théatrale ultérieure, ses réflexions théoriques sur l'art dans Die Ästhetik des Widerstands, mais aussi les procédés artistiques eux-mêmes, bref l'ensemble de son œuvre porte les traces de son travail de peintre ${ }^{1}$. Ses tentatives journalistiques d'où sont issues e.a. les Sechs Reportagen aus Deutschland pour le Stockholm Tidningen $(1947)^{2}$ restèrent une parenthèse, sans doute parce que les lois régissant ce domaine lui étaient étrangères. Il en alla tout autrement pour le film. Dans les années cinquante, Weiss ne s'est pas seulement intéressé d'un point de vue théorique au cinéma d'avantgarde, il est allé jusqu'à apprendre dans des conditions difficiles le métier de cinéaste en réalisant des films expérimentaux, des documentaires et des longs métrages. C'est sans doute le refus de prendre pour critère l'exploitation économique qui fit échouer Peter Weiss comme cinéaste et non le choix du genre en soi ${ }^{3}$. Quant à la prose à laquelle il s'essaya entre 1947 et 1959 aussi bien en suédois qu'en allemand, c'est à peine si elle fut remarquée en Allemagne ou dans son pays d'exil, la Suède, et le public n'en a pris connaissance que lorsque Weiss était déjà un auteur mondialement connu ${ }^{4}$. Il faut 
attendre l'année 1959 pour que Weiss connaisse le succès et l'estime publique avec la publication du micro-roman Der Schatten des Körpers des Kutschers publié aux Editions Suhrkamp -, certes le roman ne fut tiré qu'à 1000 exemplaires, mais il influença nombre de jeunes écrivains allemands par son écriture tout à fait inédite, basée sur l'exactitude de descriptions microscopiques. Lors de la sortie du Kutscher « Weiss était totalement inconnu en Allemagne $»^{5}$. Il ne trouva sa place définitive dans la littérature allemande qu'avec deux textes de prose le plus souvent qualifiés d'autobiographiques : Abschied von den Elteim (1961) et Fluchtpunkt (1962). Ces deux textes ainsi que Gespräch der drei Gehenden (1963) marquent un arrêt provisoire dans son travail sur la prose narrative. A partir de 1964 Peter Weiss accède à la renommée mondiale - comme auteur dramatique. Ses premières pièces (Der Turm 1949, Die Versicherung, écrite en 1952 et publiée en 1967) n'avaient eu aucun écho. Son Marat/Sade (1964) par contre fut acclamé par la critique ouest-allemande ${ }^{6}$ (à quelques exceptions près) et dans les 12 années suivantes mis en scène 85 fois entre Tokyo et Castrop-Rauxel, le Burg de Vienne et le National Théâtre de Sydney. Dans les années soixante, Weiss passa pour l'auteur dramatique allemand par excellence après Brecht. Le succès du Marat/Sade déclencha chez Weiss un processus intense d'écriture dramatique que l'on trouve souvent classé dans l'histoire littéraire sous la rubrique «Dokumentartheater»: Die Ermittlung (1965), Gesang vom Lusitanischen Popanz (1967), Viet Nam-Diskurs (1968), Wie Herrn Mockinpott das Leiden ausgetrieben wird (1968, écrit en 1963). Suivent avec Trotzki im Exil (1970) et Hölderlin (1971) deux autres drames, tandis que les années 70 sont à nouveau consacrées à la prose. C'est la naissance d'un monument : Die Ästhetik des Widerstands, parue en trois parties (1975, 1978 et 1981). En 1975 il interrompt brièvement le travail sur cet opus magnum pour se consacrer à une adaptation dramatique du Procès (1975) de Kafka, un matériau que Weiss a reprit dans le Neuer Prozeß (1982) après avoir terminé son roman, pour essayer de l'adapter au théâtre.

2 Le va-et-vient, jusqu'à la fin des années soixante, entre la peinture, l'essai, le film, la prose et l'écriture dramatique peut se concevoir par la recherche d'une reconnaissance artistique, la recherche d'un public et surtout la recherche de moyens d'existence lui permettant de subsister comme artiste. Mais à partir de 1962, et au plus tard à partir de 1964, lorsqu'il acquit une renommée mondiale, ces aspects-là ne peuvent plus entrer en ligne de compte. Se pose alors la question des motivations qui l'ont poussé à passer du roman autobiographique à l'écriture dramatique et du drame à l'écriture romanesque.

Une indication importante se trouve dans une note des Notizbücher 1960-1971 à la fin de l'année 1963: "Les nombreux et différents modes d'expression à travers lesquels j'essaie de saisir des parties de moi-même, de les interpréter - de leur trouver un dénominateur commun ${ }^{7}$. Ici comme dans d'autres textes, Weiss désigne des concepts relatifs à une catégorie artistique ou à un genre, par le mot "mode d'expression", c'est-à-dire qu'il ne part pas d'un système artistique, mais de différentes possibilités artistiques, pour exprimer ce qui jusqu'ici fut le point central de son art : la réflexion sur soi, la recherche d'intégration personnelle, d'identité. Le moi de l'auteur qui écrit ainsi se voit lui-même comme une sorte de collage, fait de morceaux inexplorés, incompris, qui n'obéissent à aucun dénominateur commun, mais qui devraient y obéir. Le dénominateur doit être élaboré à travers l'écriture. "Qui suis-je? », cette question par laquelle s'ouvre le livre préféré de Weiss, Nadja de Breton, est déjà au centre de ses premiers textes ${ }^{8}$ et en est le principe organisateur. La tonalité autobiographique, la réflexion sur soi, ne disparaissent pas avec le changement de statut, avec une position 
socialement reconnue, elle reste présente à travers l'ensemble de son œuvre. Aussi trouve-t-on le 12.12.1976 dans les Notizbücher ces remarques sur le «moi » dans Die Ästhetik des Widerstands : "Qui est ce moi ? C'est moi-même. Le livre est une recherche de moi-même "'. Ainsi dans les Notizbücher, l'auteur Weiss et le moi créé, ou plutôt réfléchi par le roman se fondent-ils pour ne plus faire qu'un : «je décris ma propre vie, je ne peux plus faire la différence entre l'invention et l'authentique - tout est authentique (comme dans le rêve tout est authentique) $»^{10}$. Ces mots ne signifient pas que tout aura été dit de la construction complexe du « moi » romanesque dans le grand roman de Weiss, mais ils sont révélateurs d'une continuité, une vie durant, de la recherche d'identité comme force motrice dans la production artistique de Peter Weiss. Certes il n'explore plus les mêmes matériaux: «L'époque était révolue où j'élaborais tout à partir de matériaux subjectifs $»^{11}$ écrit Weiss en 1965 déjà. Retenons ici que le choix des matériaux s'élargit, le monde entier, l'histoire toute entière du monde entrent dans le rayon d'action de la production artistique de Weiss, mais la recherche d'identité en restera la force motrice jusqu'à sa mort.

Il a été montré ailleurs à quel mouvement fondamental obéit la recherche d'identité de Peter Weiss dans la prose narrative jusqu'à Abschied von den Eltern: au mouvement fondamental de la verticale, d'une dynamique de l'envol triomphant et de la chute profonde. Le mouvement vertical des images que l'on retrouve dans tous les textes de prose narrative (à l'exception de Der Schatten des Körpers des Kutschers) est sémantiquement surdéterminé : sur le plan de la recherche d'identité, il désigne l'effort titanesque pour se constituer un «moi » artistique contre la maison parentale et les problèmes difficiles liés à la vie d'exilé, un effort qui est périodiquement suivi d'une dissolution, d'une décomposition du moi. Le travail sur soi s'effondre, finit dans la régression et des fantasmes de mort. La réflexion sur soi, précise jusqu'à la maniaquerie et qui à force de travail se fait écriture, finit dans le chaos, l'angoisse et le délire. L'œuvre, arrachée de haute lutte à l'informe, manque le moi de l'auteur si bien que sa dissolution dans l'informe n'est plus seulement une crainte, mais aussi un plaisir ${ }^{12}$. Dans tous les cas, la verticale qui se dessine entre l'envol et la chute, entre la recherche du moi et la perte du moi, entre l'activité artistique et l'effondrement personnel, représente l'expérience fondamentale du moi de l'auteur dans les premiers textes de prose. Tirant en quelque sorte le bilan de ces tentatives, Weiss note dans ses Notizbücher après avoir terminé Fluchtpunkt : «Plus encore qu'un témoignage sur mes délires et mes égarements qu'en fait le livre aurait dû être, il est devenu la démonstration de ma prétendue endurance et de ma force, ainsi que de l'esprit de suite de mes actions. Ces efforts pour atteindre une cohésion, un nouveau point de départ assuré font aussi partie de mon existence, mais en même temps je m'efforçais sans cesse de me cacher à moi-même les états limites où guettait la décomposition, la dissolution du moi $»^{13}$. Quelques pages plus loin dans les Notizbücher se trouvent des indications concernant le travail sur Marat/Sade, conçu à l'origine comme Hörspiel, puis repris sous forme de drame et dont la première eut lieu le 29.4.1964 au Schiller-Theater de Berlin. Le titre complet (Die Verfolgung und Ermordung Jean Paul Marats dargestellt durch die Schauspieltruppe des Hospizes zu Charenton unter Anleitung des Herrn de Sade) précise le lieu, l'action, et les protagonistes de la pièce: Weiss s'appuie sur le fait prouvé que le marquis de Sade, interné pendant la période de Restauration napoléonienne, mettait en scène des soirées de théâtre avec des patients d'un asile d'aliéné et il en fait le point de départ d'un jeu dramatique où se reflètent la Révolution française, l'ère napoléonienne et le présent. 
5 Du point de vue de la dramaturgie, la pièce est une réussite unique dans la production dramatique de Weiss. En faisant des internés d'un asile de fou les acteurs d'une pièce sur l'assassinat de Marat, il actualise sur scène la thématique de ses premiers textes de prose - décomposition de l'identité, régression, chaos, folie, torture, obsessions sexuelles, dissolution. L'abîme au bout de la verticale est toujours ouvert (comme dimension de la profondeur). Toutefois il y a un autre élément structurant dans la pièce: les grands dialogues centrés sur la conception du monde entre le révolutionnaire radical Marat et l'individualiste radical Sade.

6 Le point de vue topographique : à la ligne verticale en courbe, marquant la prose autobiographique de Weiss, se joint à présent dans le drame la ligne horizontale d'un antagonisme entre deux positions fondamentales d'égale valeur dans la conception du monde. Hegel savait déjà que « les épanchements du cœur, si l'on veut qu'ils aient une valeur dramatique, ne doivent pas se limiter à un simple travail sur des sentiments inconstants, des souvenirs et des considérations, mais qu'ils doivent rester en relation étroite avec l'action $»^{14}$ et que celle-ci est marquée à son tour par " la discorde et la contradiction entre deux états d'esprit opposés ${ }^{15}$. Seule la «Auflösung» ${ }^{16}$ exigée par Hegel, la synthèse dramatique, manque encore, et elle restera, d'un point de vue structurel, étrangère à la dramaturgie de Weiss. Certes les critiques, mais aussi les metteurs en scène, ont essayé (en partie avec le soutien actif de Weiss) de faire pencher l'équilibre dramatique tantôt en faveur de la position de Marat, tantôt en faveur de celle de Sade. A titre d'exemple, deux mises en scène célèbres: Dans celle de Peter Brook à Londres, Sade occupe une place prépondérante de par les choix d'une mise en scène inspirée du Théâtre de la cruauté d'Antonin Artaud, tandis que Perten à Rostock (où Weiss fut également présent) laisse le dernier mot à ce que représente le personnage de Marat. Weiss lui-même a essayé de déplacer le centre de gravité en procédant à de nombreux remaniements, souvent contradictoires ${ }^{17}$, mais toutes les versions ont ceci en commun qu'elles sont une mise à l'épreuve de structures de base antithétiques qui n'aboutissent jamais à une synthèse.

7 Le passage de la prose autobiographique à l'écriture dramatique (marqué par le Marat/ Sade) présuppose alors que les détours qu'emprunte le processus de la recherche d'identité chez Weiss peuvent se condenser en maintenant les antagonismes et s'objectiver dans la matière historique. Mais en même temps, les données de la prose autobiographique restent présentes sur scène. "Des problèmes personnels s'objectivent dans l'histoire $\aleph^{18}$, voilà comment Walter Jens qualifiera plus tard ce procédé. Il y va certainement de la décision que doit prendre l'individu Peter Weiss, balançant entre l'idée de la révolution politique et un hédonisme radical, empreint de scepticisme, mais il y va aussi de l'antagonisme entre l'espoir en l'avenir qui recourt à la terreur et l'affirmation d'un hic et nunc joyeux qui laisse les choses aller à vau-l'eau, d'un antagonisme donc entre une époque historique particulière et le droit au bonheur individuel, il y va en fin de compte du problème de toutes les révolutions modernes. C'est du moins ce qui peut expliquer le succès mondial d'une pièce pendant une époque de changements profonds et qui vit naître dans le tiers monde une ère révolutionnaire, anticolonialiste et socialiste.

8 "J'écris pour savoir où je suis $~^{19}$ - avec le passage de la prose à l'écriture dramatique s'est opéré un changement dans la nécessité d'écrire. Autrefois la question était : «Qui suis-je ». Et si la question s'appliquait jadis à l'existence en général, elle s'applique à présent à la conception du monde. D'où la préférence pour le mode d'expression 
particulier qu'est l'écriture dramatique et par là même pour la structure horizontaleantithétique. Cette préférence est indissociable de deux processus : premièrement de la décision de l'auteur Peter Weiss d'opter pour le point de vue socialiste, défendu officiellement en 1965 lors d'une profession de foi formulée sans la moindre équivoque : "les lignes directrices du socialisme contiennent pour moi la seule vérité valable ${ }^{20}$; deuxièmement, elle est indissociable de l'élimination progressive dans ses drames d'éléments subjectifs et autobiographiques. En 1963 déjà, Weiss prend ses distances avec le " caractère individualisé, avec ses conflits, ses crises et ses "fadaises de l'âme" " ${ }^{21}$, mais à partir du milieu des années soixante s'accumulent des prises de position où Weiss, avec le zèle du néophyte, juge sévèrement les "fadaises de l'âme » de ses premiers écrits. Sur la quatrième de couverture de la réédition de Abschied von den Eltern il fait imprimer plus tard la phrase suivante : «Je regarde cette époque comme à partir d'une autre vie, étranger au moi dont je suis issu $»^{22}$.

9 Mais de la vie de l'auteur Peter Weiss, la verticale, l'expérience de l'angoisse et de l'anéantissement de l'identité n'ont pas disparu. Ainsi peut-on lire au début de 1964 dans les Notizbücher: «Angoisse nocturne - pas de moi - pas d'identité - pas de conscience - se perdre de vue - "malade mental" - pas de volonté - pas de vouloir - pas de décision $»^{23}$. Cet état néanmoins n'apparaîtra plus dans les textes publiés pendant près de cinq ans. Weiss structurait d'ailleurs ses pièces de telle manière qu'il n'y avait plus de place pour ce matériau. Une preuve intéressante - jusqu'ici ignorée par la recherche - permettant d'étayer cette thèse se trouve dans les fragments d'un projet de Divina Comedia qui préoccupa Weiss des années durant, sans qu'il parvienne à lui donner une forme définitive et jouable. Parmi les nombreux projets se trouvait l'idée, réalisée par fragments, de faire une adaptation dramatique de Abschied von den Eltern et de Fluchtpunkt. Cette œuvre était censée être une œuvre autocritique, mais même dans cette perspective, le matériau se refusait à la forme antithétique du drame. « Le défaut esthétique de ce fragment réside avant tout dans les dialogues trop longs que l'on ne peut pas transposer sur scène $»^{24}$. La vie ne se résume pas aux « duels entre les mots d'un drame $»^{25}$ - une formulation à travers laquelle Weiss faisait allusion - en passant, mais certainement pas par hasard - à sa conception fondamentale du drame.

Weiss chassa les "fadaises de l'âme" de ses drames en plusieurs étapes. Dans Die Ermittlung, la pièce de Weiss sur Auschwitz, vu sous l'angle juridique du procès d'Auschwitz à Francfort, chasser les «fadaises de l'âme" était une vertu dictée par le sujet. La souffrance du sujet ne suffit pas à exprimer ce que fut Auschwitz. Cette folie-là n'était pas la folie d'un asile d'aliéné, la souffrance ne pouvait plus être personnifiée et ces sadiques-là n'avaient plus grand chose en commun avec Sade. Aussi paraissait-il logique que Weiss raconte dans un essai à part sa relation à un sujet qui avait déterminé sa vie. Meine Ortschaft ${ }^{26}$ rend compte d'une visite à Auschwitz, un lieu auquel il était en fait destiné.

11 Die Ermittlung est une pièce antithétique par nécessité structurelle, une pièce pour les victimes et contre les criminels qui même sur le banc des accusés restaient les vainqueurs. L'antithèse entre victime et oppresseur, entre une oppression sans cesse renouvelée et les efforts de libération hérö̈ques détermine également les deux pièces suivantes: Gesang vom Lusitanischen Popanz et Viet-Nam-Diskurs. Après une analyse approfondie de la structure dramatique du Popanz, Manfred Haiduck conclut : «Dans le Popanz aussi la construction antithétique est de nature dialectique et au service de la recherche de la vérité. Mais contrairement au Marat/Sade, et plus fortement encore que 
dans la Ermittlung, elle est régie par le point de vue du parti pris socialiste ». Pour le Viet Nam-Diskurs il arrive à une conclusion similaire : «Un moyen important d'élucider la vérité dans cette œuvre est une fois de plus l'antithèse... $»^{27}$. Pour aussi vraies que soient les remarques sur la structure antithétique, elles n'en sont pas moins problématiques quant à la prétendue « dialectique » qui dans un aveuglement héroïque ignore les indices de ce qui allait sortir de ces mouvements de libération. Parmi les vérités qu'il aurait fallu y trouver n'en fut trouvée qu'une, la plus évidente, la vérité de l'oppression colonialiste. Le Viet Nam-Diskurs représente l'ultime tentative de Peter Weiss pour évacuer du drame les "fadaises de l'âme». Mais du même coup les personnages dramatiques perdent toute individualité. Seuls parlent encore des collectifs, des couches sociales, des classes. Dans les notes sur son voyage au Vietnam, Weiss relève une remarque de son accompagnateur vietnamien à laquelle il adhère : "Thi: Très intéressant les conflits psychiques, ces récits d'un monde bourgeois décadent. La représentation des sacrifices que cela demande. Mais nous avons dépassé cela. L'individualiste souffrant est pour nous une forme démodée ${ }^{28}$.

Si on lit côte à côte les Dokumentär stücke et les notations de la même époque dans les Notizbücher, on est frappé par le rôle central de l'antithèse. Dans les pièces, elle est le principe organisateur de la structure dramatique, dans les Notizbücher elle est sujet de réflexion sans cesse renouvelé. Seulement, dans les Notizbücher, il est toujours question de contradictions internes et d'ambivalences propres à l'auteur qui écrit, il est question de cette « position entre les contradictions, entre les oppositions $»^{29}$. Ce point de vue est un préalable épistémologique à la recherche d'une vérité toujours relative ${ }^{30}$. Toutefois c'est le déchirement qui davantage encore est l'expérience existentielle de base de Peter Weiss: "Cette pensée en termes de contradictions, rongée par la maladie du doute, ... c'est comme si tout mon être était composé de deux pôles en perpétuel conflit entre lesquels tout se joue, on exige sans cesse de moi des choses contradictoires, c'est cela le moteur qui produit tout mon travail $»^{31}$. Les Dokumentär stücke sont certes organisés selon le principe des "deux pôles en perpétuel conflit ", mais ce ne sont pas les mêmes, ce sont les pôles des oppresseurs et des combattants pour la liberté, du bien et du mal en fin de compte. D'un point de vue dramatique, cette opposition n'est pas très fructueuse quand le mal est dépourvu de ce bien-fondé qui rend possible le dialogue. Sans "bien-fondé moral $\|^{32}$ des positions en conflit, d'après Hegel, pas de bouleversement par le drame, sans «bien-fondé moral », pas de possibilité de dialogue. "De la possibilité du dialogue dépend la possibilité du drame " $^{33}-$ du moins d'un drame qui mise autant sur " les duels entre les mots " que celui de Peter Weiss. Cette activité artistique autrefois développée comme mode d'expression d'un moi clivé, déchiré, devient étrangère à l'auteur : «En parlant j'entends soudain mes propres mots comme sortant d'un haut-parleur. Etrangers, ne faisant pas partie de moi ».

Les contradictions qui préoccupèrent Weiss et la structure contradictoire de ses pièces allèrent en s'accentuant dans la deuxième moitié des années soixante - au grand préjudice de ses pièces. Pendant son travail sur Viet Nam-Diskurs déjà, Weiss conçut le plan d'une pièce sur les contradictions inhérentes au socialisme d'après le modèle du Marat/Sade ${ }^{34}$. Mais en même temps, il voulait revenir au héros individuel par lequel se refléterait l'histoire de sa vie sans renoncer pour autant à l'objectivation historique. Le résultat en fut la pièce dramatique : Trotzki im Exil, écrite en 1968/69. À travers le titre déjà on peut déceler les aspects autobiographiques : l'exil auquel Trotski fut contraint par trois fois, l'exil, où, assis à son bureau, il rêvait de la révolution permanente et où il fut finalement assassiné, cet exil, Weiss l'a considéré sa vie durant comme sa forme 
d'existence propre. L'étranger, le banni, l'exclu - ce furent là des autoportraits de Peter Weiss qui s'appliquaient à la figure historique de Trotski. D'un point de vue esthétique, cette pièce qui, partant de l'exil mexicain de Trotski et par retours en arrière met en scène aussi bien les stations de sa vie que l'histoire révolutionnaire russe, est un échec total. Contrairement à Marat/Sade, sa pièce sur la Révolution française, le passé révolutionnaire n'est pas représenté comme une pièce dans la pièce, mais seulement évoqué à travers des souvenirs. De surcroit, il manque à Trotski un antagoniste, comme Marat l'avait en la personne de Sade. Cet antagoniste seul Staline aurait pu l'être, mais il n'apparaît qu'en marge. Il se peut que des raisons politiques, dramaturgiques et historiques aient empêché Weiss d'opter pour cette solution. Toutefois - comme l'a fait remarquer à plusieurs reprises Heiner Müller - il était tout aussi impossible de représenter Staline sur scène d'une manière adéquate que de mettre directement en scène Hitler ou Auschwitz. De plus la problématique du communisme mondial au vingtième siècle ne se réduit pas à l'antagonisme Trotski-Staline. Les moyens scéniques centrés sur la conception du monde empêchèrent d'autre part que Trotski puisse s'incarner sur scène dans un personnage vivant.

Quoi qu'il en soit, la pièce sur l'Antéchrist du marxisme soviétique mit Weiss dans une position intenable. En RDA et en Union Soviétique où on l'avait courtisé, il devint persona non grata, des étudiants de l'Allemagne de l'ouest perturbèrent la première de la pièce à Düsseldorf, la plupart des critiques furent négatives au point que Weiss décida de mettre fin aux représentations. "Je n'ai pas ma place dans le collectif / que faire / il n'y a pas de quoi rire " ${ }^{35}$. L'expérience de Trotzki im Exil déclencha une crise où Weiss se sentit à nouveau enfermé comme poète, acculé, expatrié. Des expériences familiales anciennes et l'expérience de l'exil semblaient se répéter. Commença alors une réflexion sur le rôle de l'art, de la vision, de l'utopie dans leur rapport à la logique du pouvoir. Hölderlin fut le prochain sujet auquel Weiss s'attaqua pour en faire un texte dramatique, Hölderlin retiré dans la folie, dans sa tour à Tübingen (Der Turm ${ }^{36}$, ainsi s'appelait le premier drame de Weiss) parce que ses espoirs dans le jacobinisme ne s'étaient pas réalisés, parce qu'il se voyait abandonné par les intellectuels de son époque.

15 Mais pendant son travail sur Hölderlin, sa santé fut sérieusement ébranlée, elle le perturba si profondément que «les fadaises de l'âme » qu'il croyait vaincues depuis longtemps le tenaillèrent à nouveau. Weiss tenta de les maîtriser en travaillant sur son Hölderlin, mais le remède éprouvé ne fonctionna pas. Weiss remarque une « résistance insurmontable $\aleph^{37}$ au travail. Le drame n'est pas en mesure d'absorber tout ce que la crise fait resurgir. D'où la création d'un journal pour la période du 10.8.1970 au 1.1.1971 qui fut retrouvé dans sa succession sous forme de manuscrit achevé et paginé. Weiss avait songé à une publication à part. Mais elle n'eut lieu qu'en 1991, neuf ans après sa mort $^{38}$. Ce texte qui jusqu'ici n'a guère suscité d'intérêt est un texte-clé pour comprendre la vie et l'œuvre de Peter Weiss.

Cet « essai d'autothérapie $»^{39}$ devait passer par une nouvelle tentative pour maitriser les «fadaises de l'âme » qu'il croyait vaincues. Dès les premières phrases du texte, le problème est posé : "Depuis des années j'ai négligé de me pencher sur mes rêves, d'approfondir mes monologues intérieurs, j'en avais fini de tout cela, tout cela je l'avais fait à outrance jadis, les événements extérieurs étaient plus importants à présent et lorsqu'il s'agissait de moi, il ne pouvait s'agir que de savoir quelle position j'occupais 
dans le monde extérieur, pour qui je prenais parti. La problématique personnelle se révélait tout au plus dans le choix de mes sujets de travail... »"

Face à une mort possible Weiss se rend compte que son écriture s'était beaucoup éloignée de sa problématique subjective qui fut si longtemps le moteur de son expression artistique. Ce qu'il s'agit de reconquérir, c'est une "masse de pensée qu'autrefois j'avais diffamée et disqualifiée pour leur romantisme, leur utopie et leur subjectivisme... $»^{41}$, une forme d'existence "où l'homme vit en harmonie avec luimême $\aleph^{42}$. Weiss tente de réaliser tout cela en notant ses rêves, souvenirs, monologues intérieurs, mais aussi en réfléchissant sur les événements politiques, des réflexions souvent déclenchées par l'actualité. Ces notes s'écrivent, contrairement au Hölderlin, "presque sans résistances, comme sous la dictée d'un monologue intérieur... » même si Weiss a parfaitement conscience que toute écriture est aussi toujours une " censure ${ }^{43}$. Et le seul fait que le rêve soit à nouveau accepté comme matériau irresponsable en soi marque une différence avec le travail artistique de Weiss les cinq années précédentes. Dans le rêve, on retrouve la proximité avec Dali, avec la tradition du surréalisme en général qui avait profondément marqué Weiss et qui avait fini par tomber sous le coup d'un verdict politique. Un regard sur le matériau - où rêves et souvenirs passent la censure d'une conscience rigoureuse $d u$ devoir politique - révèle l'expérience existentielle fondamentale de l'auteur Peter Weiss et les structures fondamentales de toute son œuvre.

Il y a là l'expérience fondamentale de l'horreur et la protection qu'offre la mère contre cette horreur : « ... et je voyais ma mère couchée à côté de moi au lit, je n'avais jamais pris congé d'elle, elle vivait encore, elle était couchée à ma droite... $\star^{44}$. Il y a là le désir d'être reconnu pour son travail artistique par le père réel, un élément onirique que Weiss, dans l'interprétation qu'il fait de ses rêves, omet de voir, comme par hasard ${ }^{45}$. Il $\mathrm{y}$ a là son adhésion au modèle " romantique, étranger au monde $»^{46}$ que représente son père spirituel Hermann Hesse. Il y a là son adhésion au monde insouciant "des prostituées, des joueurs, des artistes du cirque, des comédiens, ... des exclus, des marginaux, des naufragés d'un drôle de milieu, des enfers, une sorte de royaume des morts qui n'avait rien en commun avec le royaume des ombres, mais au contraire permettait des rencontres et des émotions... $»^{47}$. Le plaisir qui fait fi de tout engagement social resurgit dans un texte de Weiss - dans le lit de sa mère, ou celui d'une prostituée. Le plaisir et la mort. Cinq stations de sa vie - tantôt rêvées, tantôt évoquées par le souvenir - où Weiss séjourna aux confins de la mort. À l'expérience de la mort prochaine viennent s'ajouter des rêves de fuite et de persécution.

D'un point de vue topographique, Weiss retrouve avec Rekonvaleszenz la verticale qui avait été la marque de ses textes jusqu'au Marat/Sade $e^{48}$. Il y a aussi le retour de la courbe entre la lutte pour la forme et le plaisir à dissoudre ce qui vient d'être formé ${ }^{49}$. Cela vaut surtout pour les récits des rêves et des souvenirs. Les textes commentant les événements politiques restent par contre empreints d'un parti pris socialiste, voire d'un "sens de la justice jusqu'à l'excès $»^{50}$. Les aspects politiques n'ont donc pas disparu, mais ils sont mis en rapport avec des expériences autobiographiques et les lieux de leur conservation : le rêve et le souvenir.

21 En même temps, le rayon de la réflexion politique s'élargit. La foi emphatique en l'avenir, exprimée dans les deux derniers Dokumentärstücke, cède la place à la possibilité que "l'ère révolutionnaire touche à sa fin " $^{11}$. Mais ce sont surtout les limites des "modèles didactiques" documentaires, tels que Weiss les avait mis en scène, qui 
apparaissent clairement : «Si je sélectionne un événement ici ou là et si j'essaie de le représenter dans ses justes proportions, dans son juste contexte, je suis tôt ou tard gagné par un sentiment d'impuissance, un sentiment d'impuissance qui doit prendre le dessus, car la complexité ne peut plus être maîtrisée par un seul, elle est maîtrisée par des super-cerveaux électroniques, et je n'ai qu'un seul réconfort, celui d'avoir au moins produit un modèle didactique à l'usage de débutants comme moi, primitifs, ridiculement petits par rapport à l'objet... $»^{52}$. Reste alors la technique d'objectiver à travers des personnages scéniques les positions contradictoires de l'auteur et de sa réflexion, mais il existe à présent une quantité de couples contradictoires: la technique, de linéaire et antagoniste, se fait " prismatique ». "Transférer cette épreuve de force parfois insupportable à une multitude de locuteurs qui intègrent à travers les différents rôles et éclairent comme à travers un prisme cette problématique insoluble pour moi, est une thérapie permettant de surmonter les difficultés que rencontre tout individu voulant obtenir des résultats tangibles. En transposant sur scène les couples contradictoires ancrés en moi, je peux alors regarder ma manie du doute avec bonne conscience, et tout ce qui d'habitude fait qu'en écrivant le sol se dérobe sous mes pieds, prend alors consistance et force de conviction $»^{53}$.

Retour aux expériences existentielles de jeunesse qui avaient marqué les premiers écrits; conserver les rêves, les visions, les souvenirs comme moyens d'expression; continuer de prendre parti pour les opprimés contre les oppresseurs ; garder un espace de réflexion pour la matière historique dont la complexité interne et la contradiction sanglante s'imposent toutefois davantage à la conscience - ce programme d'écriture de plus en plus complexe ramène Weiss à l'écriture narrative. La crise de Rekonvaleszenz marque le point où sont ramenés à la conscience, pour devenir texte, des expériences et des désirs individuels et refoulés, des contradictions refoulées; elle marque aussi le passage de la production dramatique des années soixante à la Ästhetik des Widerstands.

Dans Ästhetik des Widerstands, le passé de l'auteur n'est pas rejeté, il renoue plutôt avec " la continuité qui pour moi se perd dans un grand brouillard, mais qui néanmoins est présente quelque part... $»^{54}$. Dans le brouillard, se dessinent avec une clarté étrange des images obsessionnelles, centrées surtout sur des métaphores de vol et de chute ${ }^{55}$. Elles sont à mettre en rapport avec la perspective antifasciste dans Die Ermittlung, avec l'éloge du travail politique et le doute profond vis-à-vis de ce travail tel qu'il s'exprime dans Marat/Sade, avec la réflexion sur les contradictions internes au socialisme dont traite la pièce sur Trotski; à mettre en rapport aussi avec le retour d'une foi en la puissance du rêve, des visions et de l'art qui gardent leur autonomie par rapport à la politique. Weiss ne renonce pas au procédé qui caractérise ses grands drames : faire parler des oppositions, des antagonismes, par ex. l'opposition entre le père du moi narrateur - qui tente de conjurer en les nommant les crimes inconcevables des fascistes - et la mère qui, face au génocide dont elle soupçonne l'existence, sombre dans le mutisme de la dépression. Ou encore, celle entre les deux amis du moi, Coppi et Heilmann, l'un défendant la nécessité d'une politique pratique, l'autre prônant sa transgression visionnaire. Mais le roman peut déployer une multitude de niveaux

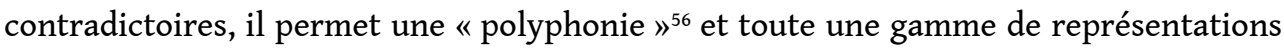
narratives dont ne dispose pas le drame ${ }^{57}$. Les deux drames que Weiss écrivit pendant et immédiatement après son travail sur Die Ästhetik des Widerstands - deux adaptations du Procès de Kafka - montrent clairement que la complexité de ses intentions littéraires 
pendant les années soixante-dix excédait largement les possibilités de la forme dramatique dont il disposait.

Dans une brève Évocation de Peter Weiss, Heiner Müller a placé Die Ästhetik des Widerstands au-dessus des drames, et a assigné à ceux-ci, très pertinemment, un lieu historique : «Parler du Vietnam signifiait taire Bautzen. Peter Weiss vivait encore - tout comme Brecht jusqu'à sa mort survenue à temps - dans deux mondes, l'un d'eux était imaginaire; nous, nous habitions un tiers monde où le plus petit collectif était le schizophrène... Ce qui reste, c'est l'auteur d'une œuvre qui a marqué son siècle, une autre recherche du temps perdu $»^{58}$. Néanmoins, l'immense, l'incommensurable effort d'écrire un grand roman n'a pas permis à Weiss de réaliser cette "unité ${ }^{59}$, cette " harmonie avec lui-même ", telles qu'il les recherchait en tant qu' artiste et citoyen. L'unité du sujet n'était pas faisable: «Nous trimballons sur nos deux jambes l'hypothèse d'une personnalité, à cette personnalité nous avons donné du travail, elle est employée chez nous, nous l'alimentons en nourritures spirituelles, préparées sur un foyer sous lequel fait rage le feu d'un sombre, d'un terrible égarement $»^{60}$.

\section{NOTES}

1. Aussi peut-on lire dans les Notizbücher 1971-1980: "Être peintre avant tout, bien que les images contiennent quantité d'associations de l'esprit, d'antagonismes psychologiques, philosophiques et politiques. Ce sont précisément ces couleurs-là, ce jeu déformes-là qui peuvent les exprimer, et non la lourdeur du poème, l'essai savant, les duels entre les mots dans les drames. - La violence du mouvement est là, l'expressivité des gestes et le labyrinthe tortueux des idées, mais il y a là le silence, le silence de la peinture. Ce ne sont pas des déroulements d'événements compliqués qui sont représentés, mais des états, des états qui contiennent des épopées entières, silencieuses, et qui entrent en mouvement dans le volume de pensée du spectateur pour le conduire à sa propre manière de vivre la crise de son époque. " traduit par l'auteur d'après : Peter Weiss, Notizbücher 1971-1980. Francf./M, 1981, p. 120.

2. In : Peter Weiss, Die Besiegten, Francf/M. 1985, p. 125-153.

3. Manfred Karnick a montré que la pratique dramatique de Weiss est largement déterminée par son travail de théoricien dans le cinéma d'avant-garde. Manfred Karnick: «Peter Weiss' dramatische Collagen. Vom Traumspiel zur Agitation.» In : Peter Weiss (sous la direction de Rainer Gerlach), Francf/M. 1984, p. 220.

4. Von Insel zu Insel, Berlin 1984 (édition originale Stockholm 1947) ; Die Besiegten, Francf. /M.1985 (édition originale Stockholm 1948; Der Fremde, publié à Franc. /M en 1980 sous le pseudonyme Sinclair (édition originale 1949); Das Duell (édition originale Stockholm 1953).

5. Urs Jenny : «Abschied von den Eltern», in, Über Peter Weiss (sous la direction de Volker Canaris), $4^{\mathrm{e}}$ éd. Francf. /M. 1976, p. 46.

6. Cf. l'étude de Jochen Vogt : Peter Weiss, Reinbek bei Hamburg 1987, p. 84 s.

7. Peter Weiss, Notizbücher 1960-1971, Francf./M. 1982, p. 120.

8. «Wer bin ich» Peter Weiss, Die Besiegten, op. cit., p. 120.

9. Op. cit., p. 539.

10. Op. cit., p. $872 \mathrm{~s}$.

11. «Interview avec Peter Weiss » in, Theater heute, $n^{\circ} 10,1966$, p. 14. 
12. Karl Heinz Götze, Poetik des Abgrunds und Kunst des Widerstands. Grundmuster der Bildwelt von Peter Weiss. Opladen 1995, p. 85-100 et 152-204.

13. Notizbücher, op. cit., p. $96 \mathrm{~s}$.

14. Traduit d'après Hegel : Vorlesung über die Ästhetik. Band 3. In, Hegel, Werke in zwanzig Bänden, Bd. 15, Francf./M 1970, p. 490.

15. Hegel, op. cit., p. 488.

16. Ibid.

17. Cf. Manfred Haiduck : Der Dramatiker Peter Weiss, $2^{\mathrm{e}}$ édition, Berlin 1977, p. 104.

18. In, Die Zeit du 29.3.1968.

19. Réponse de Peter Weiss à une enquête de la revue Theater heute, août 1963. in, Materialien zu Peter Weiss'Marat/Sade, sous la direction de Karlheinz Braun, Francf./M. 1967, p. 99.

20. Peter Weiss : «10 Arbeitspunkte eines Autors in der geteilten Welt», in, Weiss, Rapporte 2, Francf./M. 1971, p. 22.

21. Frankfurter Rundschau du 24.8.1963.

22. Francf./M. 1980.

23. Weiss, Notizbücher, t. 1, p. 218.

24. Cf. Manfred Haiduck qui a eu accès très tôt à ce fragment. MH., Der Dramatiker Peter Weiss, op. cit., p. 121

25. Weiss, Notizbücher, t. 2, p. 120.

26. In, Rapporte, op. cit., pp. 113-125.

27. Haiduck, Peter Weiss, op. cit., p. 161 et p. 183.

28. Notizbücher 1960-1971, p. 587.

29. Ibid., p. 225.

30. «Il n'y a pas pour moi de vérité univoque. La possibilité (l'hypothèse) de la vérité naît de doutes et de contradictions. » Ibid., p. 356.

31. Ibid., p. 850.

32. Hegel, Ästhetik, op. cit., t. 3, p. 525.

33. Peter Szondi : Theorie des modernen Dramas (1880-1950), Francf./M. 1959, p. 19.

34. "Le problème Marat/Sade transposé à l'antagonisme à l'intérieur du socialisme : le marxiste, pour qui tout est politique, et le reste sclérosé, fané, le marxiste qui n'a pas de vie privée - contre le révolutionnaire pour qui tout est mouvement, fantaisie, espoir - utopie aussi -. » Ibid., S.536.

35. Ibid., p. 594.

36. Stuttgart 1974, écrit en 1949.

37. Peter Weiss, Rekonvaleszenz, Francf./M. 1991, p. 8.

38. Pour la genèse éditoriale, cf. op. cit., p. 217.

39. Ibid., p. 124.

40. Ibid., p. 7

41. Ibid., p. 8 .

42. Ibid., p. 213.

43. Ibid., p. 124.

44. Ibid., p. 108.

45. Cf. ibid., p. 12.

46. Ibid., p. 111.

47. Ibid., p. 9.

48. Pour l'analyse détaillée cf. Götze, Poesie des Abgrunds, op. cit., p. 182-195.

49. «Pour moi tout naît de l'informe, de l'inconscient, de l'onirique, ce n'est qu'après que le premiers mots ont été extraits de tout cela, que sont retenus et élaborés de longs enchaînements d'associations d'idées, mais quand enfin le sujet est épuisé, l'incertitude reprend le dessus, et à ce moment là, je préférerais restituer le tout à un univers ouvert, à l'inachevable, au domaine des infinis possibles... » Weiss, Rekonvaleszenz, op. cit., p. 183. 
50. Ibid., p. 159.

51. Ibid., p. 193.

52. Ibid., p. 160.

53. Ibid., p. 170.

54. Weiss, Notizbücher 1971-1980, op. cit., p. 872.

55. Cf. par ex. Peter Weiss, Abschied von den Eltern, op. cit., p. 17, Die Ästhetik des Widerstands, t. 1, p. 90 sq ou encore Abschied, op. cit., p. 52. Die Ästhetik des Widerstands, p. 90.

56. Cf. Michail Bakhtin, «Das Wort im Roman», in Die Ästhetik des Wortes, Francf./M. 1979, p. 155 sq.

57. Peter Szondi, Theorie des modernen Dramas, op. cit., p. 17.

58. In Peter Weiss, Leben und Werk, sous la direction de Gunilla Palmstierna-Weiss et Jürgen Schutte, Francf./M. 1991, p. 22 et 23.

59. Peter Weiss, Rekonvaleszenz, op. cit., p. 213.

60. Peter Weiss, Notizbücher 1971-1980, op. cit., p. 872.

\section{RÉSUMÉS}

« Recherche d'identité et théâtre politique » retrace les diverses tentatives artistiques (peinture, journalisme, cinéma, prose romanesque et autobiographique, théâtre) de Peter Weiss pendant les années soixante et soixante-dix pour montrer comment le choix du genre s'articule autour de la problématique de l'identité qui se cherche dans l'engagement politique et dans d'autres cheminements. Quelle écriture exprime le mieux l'antagonisme et la contradiction qui caractérisent l'œuvre entière de Peter Weiss mais aussi tout son être ? Tel est le questionnement de cette étude.

«Identitätssuche und politisches Theater» analysiert die verschiedenen künstlerischen Versuche Peter Weiss' in den sechziger und siebziger Jahren (Malerei, Publizistik, Film, Roman und Autobiographie, Theater), um aufzuzeigen, wie die Wahl des Genres um eine Identitätsproblematik zentriert ist, in der die eigene Identität im politischen Engagement und auf anderen Wegen gesucht wird. Welche Schreibweise bringt mit der größtmöglichen Präzision den Antagonismus und den Widerspruch zum Ausdruck, die das gesamte Werk des Peter Weiss aber auch sein ganzes Wesen kennzeichnen? Auf eine solche Fragestellung möchte diese Studie näher eingehen.

\section{AUTEUR}

\section{KARL HEINZ GÖTZE}

Université d'Aix-en-Provence 